Relations industrielles

Industrial Relations

\title{
La citoyenneté au travail ?
}

Une introduction

\section{Towards Citizenship at Work?}

\section{An Introduction}

\section{Michel Coutu et Gregor Murray}

Volume 60, numéro 4, automne 2005

La citoyenneté au travail ? Réflexions sur le milieu de travail de

l'avenir

Citizenship at Work? Thinking the Workplace of the Future

¿La ciudadanía laboral? reflexiones sobre el medio laboral del futuro

URI : https://id.erudit.org/iderudit/012337ar

DOI : https://doi.org/10.7202/012337ar

Aller au sommaire du numéro

Éditeur(s)

Département des relations industrielles de l'Université Laval

ISSN

0034-379X (imprimé)

1703-8138 (numérique)

Découvrir la revue

\section{Citer ce document}

Coutu, M. \& Murray, G. (2005). La citoyenneté au travail ? Une introduction. Relations industrielles / Industrial Relations, 60(4), 601-630.

https://doi.org/10.7202/012337ar

Tous droits réservés (C) Département des relations industrielles de l'Université Laval, 2005
Ce document est protégé par la loi sur le droit d'auteur. L’utilisation des services d'Érudit (y compris la reproduction) est assujettie à sa politique d'utilisation que vous pouvez consulter en ligne.

https://apropos.erudit.org/fr/usagers/politique-dutilisation/ 


\title{
La citoyenneté au travail? Une introduction
}

\author{
Michel Coutu \\ Gregor MurRay
}

Dans son étude fondatrice «Developping Industrial Citizenship : A Challenge for Canada's Second Century », Harry Arthurs (1967) célébrait avec optimisme la longue marche des travailleurs vers la pleine jouissance des droits civils, politiques et sociaux dans les sociétés industrielles avancées. Sa lecture des événements fournissait un récit hautement stimulant de l'évolution passée et présente du droit du travail au Canada, culminant dans l'affirmation graduelle mais inévitable de la citoyenneté industrielle. T.H. Marshall (1949) avait déjà analysé le développement des différentes dimensions de la citoyenneté dans son étude, devenue depuis incontournable, des rapports entre la structure économique de l'Angleterre et les droits fondamentaux reconnus à ses citoyens à partir du $18^{\mathrm{e}}$ siècle. Bien que la citoyenneté n'avait pas encore atteint son plein épanouissement, ses traits principaux apparaissaient manifestes, du fait à la fois de l'émergence de l'État-providence dans l'après-guerre et, suivant la formule de Otto KahnFreund (1954), de la maturité croissante de «l'autonomie collective » en droit collectif du travail. Arthurs assuma une vision similaire et se fit l'écho de l'optimisme ambiant de l'époque en anticipant le développement apparemment inévitable et nécessaire des droits collectifs dans la sphère économique, ce qu'il qualifia de « citoyenneté industrielle ». Kerr, Dunlop, Harbison et Myers (1960) envisageaient alors le pluralisme industriel comme forme de stabilisation des sociétés industrielles du point de vue de la sphère du travail, les revendications légitimes en conflit se transformant en normes consensuelles fondées sur les droits collectifs reconnus aux travailleurs : c'est bien de manière similaire que Arthurs concevait la trajectoire future des politiques publiques canadiennes en matière d'emploi et de droit des rapports collectifs du travail.

- Coutu, M. et G. Murray, École de relations industrielles, Université de Montréal et Centre de recherche interuniversitaire sur la mondialisation et le travail (CRIMT - Université de Montréal, Université Laval, HEC Montréal). 
Cette vision demeurait fonction d'une cristallisation des droits collectifs du travail. Sous-tendue par le pluralisme des acteurs collectifs sur les lieux (industriels) de travail, elle s'intégrait au cadre plus large de l'ensemble des droits économiques et sociaux vers lequel les sociétés industrialisées devaient, et allaient en toute hypothèse, évoluer. De fait, cette vision traditionnelle de la citoyenneté industrielle reposait sur une perspective univoque : celle de l'extension graduelle de la sphère des droits et libertés des travailleurs, d'abord via la reconnaissance des droits politiques, avec l'octroi du suffrage universel vers le début du $20^{\mathrm{e}}$ siècle ; ensuite, par l'affirmation des droits collectifs du travail comme contrepoids au droit de propriété, par exemple de la liberté positive d'association aux fins de la négociation collective des conditions de travail et de la promotion de la syndicalisation ; enfin, par la garantie des droits sociaux (sécurité sociale, assurance-maladie, droit à l'éducation) liés à l'émergence de l'État social dans l'immédiat après-guerre. Dans le domaine des relations industrielles, l'essor d'une citoyenneté effective pour les travailleurs fut le produit tant de la négociation collective que de l'intervention de l'État en matière de droits individuels et collectifs.

Après coup, on ne peut qu'être frappé du caractère paradigmatique de cette vision du futur résumée par la notion de « citoyenneté industrielle » pour le champ des relations industrielles, du droit du travail et des politiques publiques en matière de travail. De ce paradigme formant consensus dans la communauté scientifique découlaient les prémisses suivantes : le travail ne constitue pas une commodité, il demeure le fruit de l'activité humaine et cette caractéristique, au besoin en opposition aux opérations marchandes, doit être reconnue par le biais des normes, règles et procédures édictées par les acteurs sociaux légitimes du champ des rapports collectifs de travail. Non seulement l'idée de citoyenneté industrielle devint l'étalon normatif jaugeant les avancées des rapports de travail dans les différents systèmes industriels, mais encore elle influença de manière croissante les instruments internationaux relatifs aux droits fondamentaux de la personne. Le texte d'Arthurs représentait par conséquent un moment décisif dans l'idéalisation des relations de travail futures qui fut celle des acteurs de l'époque, et de ce point de vue il conserve tout son intérêt. Toutefois, la plupart des observateurs contemporains, à commencer par Arthurs lui-même (1996 et 1999), sont maintenant d'accord pour dire que les événements ont pris finalement une tournure fort différente.

Les milieux de travail au Canada et ailleurs se sont profondément transformés au cours des dernières décennies et le rythme des changements paraît toujours s'accélérer. Parmi les facteurs qui conditionnent ces transformations, mentionnons le développement des technologies de l'information et de la communication (Castells, 1996), l'internationalisation croissante 
de la production des biens et services (Held, 1995), les changements dans l'organisation du travail et de la gestion de la production (Murray et al., 2002), la déstructuration et la restructuration du travail dans le temps et dans l'espace (Stone, 2004), enfin la multiplication et la fragmentation des identités sociales, individuelles et collectives, au travail et ailleurs (Francfort et al., 1995, Piore, 1995). L'étendue des changements conduit en outre à un «découplage » croissant entre le cadre institutionnel du travail et de l'emploi (et ses présupposés), d'une part, et les transformations en cours qui bousculent le monde du travail, d'autre part.

La notion de citoyenneté industrielle demeure ancrée dans un modèle de production suranné et repose sur des prémisses qui se voient toujours davantage remises en question. Les travailleurs concernés par les droits collectifs du travail s'inséraient dans un univers à prédominance masculine, celui du marché formel du travail avec comme modèle l'emploi permanent à temps complet, à l'opposé du travail domestique non rémunéré assumé par les femmes et voué au «prendre soin » (care) et à la reproduction sociale (Fudge et Vosko, 2001). Dans ce schème de l'emploi industriel bénéficiant de la permanence, on présumait que la masse des salariés se verrait représentée collectivement par les organisations syndicales. Toutefois, les bouleversements en cours quant à la répartition de l'emploi, la réorganisation du travail en direction d'unités fragmentées et le déclin relatif de la représentation collective soulèvent des questions importantes en regard de la qualité du travail et de la représentation collective. En tout état de cause, il n'est plus suffisant de parler tout simplement de «citoyenneté industrielle», ne serait-ce que parce que l'emploi est de moins en moins limité à l'industrie. Plus encore, ces transformations soulèvent la question des frontières du domaine de l'emploi, en regard du rôle des femmes dans la reproduction sociale, hors les cadres du marché formel du travail.

De manière similaire, l'affirmation croissante des identités individuelles et collectives (en fonction du sexe, de l'ethnicité, de l'orientation sexuelle, du type de profession, etc.) conduit à mettre l'accent sur une variété différente de droits, en particulier les droits à l'égalité, ce qui emporte des conséquences de tout premier plan pour le droit des rapports collectifs du travail. Alors que le droit collectif du travail offrait autrefois un paradigme englobant et même autosuffisant pour la compréhension de la citoyenneté au travail, l'émergence des nouveaux référents identitaires et la multiplication des instances vouées à leur promotion, interprétation et mise en œuvre mettent ce paradigme traditionnel à rude épreuve. Les interprétations institutionnelles divergentes de la citoyenneté au travail apparaissent tantôt compléter ce paradigme traditionnel fondé sur les droits collectifs du travail, tantôt entrer directement en conflit avec lui (Vallée, Coutu et Hébert, 2002). 
Enfin, la redéfinition du rôle de l'État dans plusieurs pays industrialisés a aussi pour effet de remettre en question les conceptions prédominantes en matière de sécurité sociale et économique, en opposant la notion de responsabilité individuelle à celle de droit collectif. À cet égard, les accords institutionnels qui déterminent la citoyenneté industrielle reflètent les compromis passés entre la classe ouvrière et ses organisations et l'État, lesquels n'ont plus du tout la même portée. Au surplus, les droits collectifs qui découlent de ces accords étaient basés sur des modes de gouvernance limités au territoire national et non sur les notions transversales des droits humains et de la citoyenneté globale.

L'expression d'un large spectre d'identités individuelles et collectives sur le plan socioprofessionnel, l'introduction de nouveaux modèles organisationnels de production des biens et services et la mondialisation croissante des relations économiques et sociales ouvrent ainsi un vaste espace de recherche visant à redéfinir adéquatement la citoyenneté au travail dans le cadre de la mondialisation.

Tel est le point de départ de ce numéro spécial de la revue Relations industrielles/Industrial Relations. En faisant place à des contributions inédites fondées sur une grande diversité de perspectives, il vise à mettre en lumière un certain nombre de pistes de réflexion sur le thème de la citoyenneté au travail. À l'initiative du projet « Repenser les institutions du travail et de l'emploi à l'ère de la mondialisation » des Grands travaux de recherche concertés du Conseil de recherches en sciences humaines du Canada (GTRC-CRSHC) et du Centre de recherche interuniversitaire sur la mondialisation et le travail (CRIMT - Université de Montréal, Université Laval, HEC Montréal), et en coopération avec un grand nombre de partenaires du monde du travail et du secteur gouvernemental, un colloque sur le thème de la «Citoyenneté au travail : repenser les milieux de travail du futur» a été organisé par le CRIMT à l'Université Laval en juin 2004². Des invitations spécifiques furent lancées (tant dans le milieu universitaire qu'auprès des partenaires) aux experts de la question, en même temps qu'un appel à

1. Le support financier, matériel et organisationnel nécessaire à la tenue de ce colloque a été le fait du Conseil de recherches en sciences humaines du Canada, du Fonds québécois de recherche sur la société et la culture, de Développement des Ressources humaines Canada (Programme de partenariat patronal-syndical), de Développement économique et régional de recherche, du ministère du Travail, du Bureau international du Travail, du Centre patronal et syndical du Canada, des Réseaux canadiens de recherche en politiques publiques, de la revue Relations industrielles/Industrial Relations, ainsi que de l'Université Laval et de son Département des relations industrielles. Nous remercions en particulier Judith Paquet et Nicolas Roby pour le travail fait dans le cadre du comité d'organisation scientifique ainsi que Christan Brunelle, Jean Charest et Pierre Verge dans la sélection initiale des appels de communication et les évaluateurs anonymes dans le cadre de ce numéro spécial. 
contribution circulait dans la communauté scientifique. Les propositions de communication reçues furent ensuite soumises à un processus d'arbitrage. Les articles qu'on lira dans le présent numéro sont ceux qui ont fait l'objet d'un examen attentif par des évaluateurs externes².

Dans la suite de cette introduction, nous identifions et discutons de certaines des pistes de réflexion qui émanent de cet ensemble d'études, diversifié et riche de contenu.

Le concept de citoyenneté, tout en ayant une grande force évocatrice car il interroge les fondements même de la démocratie et de l'État, demeure passablement flou (Schnapper, 1997 : 73). Lorsque rapporté au domaine du travail, la notion est encore plus problématique. De multiple concepts dérivés sont utilisés sans que leur signification ne soit définie de manière suffisamment précise. Par exemple, on se référera à la citoyenneté industrielle, à la citoyenneté sociale, à la citoyenneté de ou dans l'entreprise, à la citoyenneté globale, etc. Un travail de clarification conceptuelle apparaît indispensable, en partant du concept premier de citoyenneté, pour considérer ensuite la problématique de la citoyenneté au travail.

Le concept de citoyenneté est utilisé dans un sens descriptif ou normatif/prescriptif, et fréquemment en entremêlant ces deux dimensions, qu'il importe pourtant de dissocier du point de vue analytique. Souvent, l'idée de citoyenneté est lancée pour exprimer un projet, un idéal, voire des aspirations plus ou moins utopiques : par exemple, l'idée d'une citoyenneté supra-nationale chez Habermas (1996, 2000). Mais fréquemment aussi, on se réfère à la notion de citoyenneté pour décrire un état de fait, un ensemble de phénomènes sociaux : tel est le cas chez T.H. Marshall (1949) ou, plus récemment, chez Dominique Schnapper $(1994,2002)$. Même si des choix de valeurs conditionnent, comme le relèvent plusieurs critiques, certains éléments de l'analyse, l'intention clairement exprimée chez ces auteurs est de retracer de manière sociologique le parcours de la citoyenneté, et non de faire ouvre de philosophie politique ou morale.

À ces dimensions descriptives et normatives, nous croyons nécessaire d'ajouter un autre axe de démarcation, suivant les intérêts de recherche mobilisés : encore que ces deux pôles puissent être entrelacés, on décèle, dans l'ensemble des travaux portant sur la citoyenneté, un intérêt primordial porté vers le statut de citoyen ou, à l'opposé, vers son identité (Hassenteufel,

2. Les contributions des collaborateurs invités doivent être pour leur part publiées sous l'égide du CRIMT dans les ouvrages suivants : Travail et citoyenneté : quel avenir ?; Broken Paradigms : Rethinking Citizenship at Work in a Global Era. 
1996). Dans le premier cas (ce que nous appellerons la «citoyennetéstatut »), la citoyenneté est abordée en référence au statut socio-juridique dont bénéficie un groupe d'individus, en fonction des droits fondamentaux (civils, politiques, sociaux, culturels) qui lui sont reconnus. Dans le second cas, l'intérêt de recherche se porte vers un problème fort différent, qui est celui de l'identification fondée sur un sentiment d'appartenance plus ou moins fort qui relie un individu ou un groupe d'individus à une entité déterminée, normalement l'État, mais tout aussi bien une communauté religieuse, ethnique, linguistique ou culturelle. Nous parlons en ce second cas de « citoyenneté-identité ». Relèvent clairement de la citoyennetéstatut les travaux de T.H. Marshall, fondés sur la distinction entre les droits fondamentaux (basic rights) civils, politiques ou sociaux reconnus historiquement aux citoyens anglais. Par contre, l'intérêt de Schnapper se porte prioritairement vers la citoyenneté-identité, parce qu'elle s'interroge essentiellement sur les conditions d'existence d'une « citoyenneté politique » qu'elle estime indispensable au maintien du lien social entre les membres de la «communauté des citoyens ».

À des fins de clarification conceptuelle, nous posons donc deux axes, allant de l'identité au statut, et du normatif au descriptif. En fonction de l'accent mis sur l'une ou l'autre de ces quatre dimensions, il est possible de départager l'ensemble des travaux portant sur la citoyenneté. Par exemple, la réflexion d'Habermas sur la citoyenneté est axée vers le normatif et l'identitaire, alors que la conception de T.H. Marshall se pose dans l'espace du descriptif et du statutaire.

Doit-on privilégier l'une ou l'autre de ces dimensions, et en exclure certaines d'une réflexion rigoureuse portant sur la citoyenneté et, plus spécifiquement, sur la citoyenneté au travail ? Là n'est pas la question, à notre avis. Toutes ces dimensions sont utiles à la compréhension de la problématique de la citoyenneté au travail et de la dynamique des discussions qui s'y rattachent. Toutefois, il importe absolument d'être conscient de ces différents niveaux d'analyse.

S'y greffe en plus un autre degré de complexité, d'où la richesse des textes dans ce numéro spécial. Si le sens de la citoyenneté n'est pas facilement arrêté, c'est parce qu'il est lui-même le plus souvent en mouvement - l'objet d'une négociation continue entre les acteurs sociaux. Le traitement évolutionniste de Marshall met en valeur l'octroi progressif des droits fondamentaux (basic rights) et la manière avec laquelle l'égalité formelle des droits civils entre en conflit avec les réalités socio-économiques des classes sociales : d'où l'importance de la reconnaissance des droits sociaux et la recherche de nouvelles formes, plus significatives, d'égalité. La démarche d'Harry Arthurs, évoquée précédemment, nous sert aussi de point de référence. Dans son article fondateur de 1967, Arthurs s'intéresse 
avant tout à la citoyenneté comme statut, comme ensemble de droits conférés à un groupe de travailleurs - encore que la dimension identitaire ne soit pas complètement absente. Par ailleurs, la posture méthodologique d'Harry Arthurs se veut essentiellement descriptive et explicative. Mais, comme l'a reconnu l'auteur par la suite, son dessein allait bien au-delà d'un pur constat sociojuridique : l'étude de 1967 exprimait aussi un idéal, esquissait un projet d'épanouissement de la citoyenneté au travail (Coutu, 2004), - projet qui, on peut le dire rétrospectivement, a tourné court, pour les raisons déjà mentionnées. Pour l'exprimer ainsi, c'est la tension entre l'être et le devoir être qui nous fournit une carte routière pour lire l'évolution des politiques publiques à l'égard du travail.

Il en va de même pour la tension entre le statut et l'identité. Nous avons justement évoqué l'importance de la prolifération et de l'affirmation des identités qui touchent le travail. Ces identités trouvent-elles reconnaissance sur le plan des statuts? De fait, nous y retrouvons un des vecteurs de la recherche de l'égalité sous l'égide des chartes des droits. À bien des égards, nous faisons face ainsi à un laboratoire de négociation entre le statut et l'identité. Stasiulis et Bakan (2005), par exemple, fournissent une étude palpitante de la négociation de la citoyenneté de la part des femmes migrantes. Dans ce cas précis, des identités diverses réclament la reconnaissance d'un statut, à plusieurs titres (femmes, travailleuses, membres d'une nation) mais cette reconnaissance suppose la mobilisation des acteurs, un processus qui est loin d'être achevé et dont les résultats demeurent indéterminés.

Autrement dit, l'intérêt des discussions autour de la citoyenneté au travail découle de la compréhension des tensions entre ce qui est et ce qui devrait être, entre l'affirmation des identités et la reconnaissance ou non de leur statut. Le fait que les milieux de travail soient bouleversés par le changement, les écarts manifestes entre l'univers du vécu et l'univers du possible nous amènent vers un immense chantier de réflexion et de négociation de la citoyenneté au travail par les acteurs sociaux eux-mêmes, tant sur le plan identitaire et statutaire que dans les sphères normative et descriptive. C'est justement le sens de l'axe du programme de recherche du CRIMT portant sur la citoyenneté au travail, du colloque portant sur ce même thème et de ce numéro spécial de RI/IR. Portons maintenant notre regard sur le contenu de ce numéro spécial.

Dans une lecture saisissante de l'évolution de la citoyenneté au travail, Judy Fudge estime que la notion de « citoyenneté industrielle », telle que conçue depuis la fin du second conflit mondial, est sujette à une période d'érosion. En se référant aux travaux de Harry Arthurs (1967), Judy Fudge 
définit la citoyenneté industrielle comme un statut limitant la chosification des salariés, la possibilité de les considérer comme une marchandise : ce statut repose sur toute une gamme de droits individuels et collectifs, et son essor est inextricablement lié à celui de l'État social interventionniste. Retraçant l'historique du régime de la citoyenneté industrielle au Canada, Judy Fudge en montre la portée limitée (au regard des femmes en particulier) et la faible institutionnalisation. À l'origine, la demande explicite d'une citoyenneté industrielle reposait sur la reconnaissance de la liberté syndicale et sur l'institution de la négociation collective. Il ne faut pas perdre de vue, par ailleurs, que le titulaire de la citoyenneté industrielle était le pourvoyeur mâle, indifférent, tout comme les syndicats de l'époque, à la promotion des droits de la femme. Les années 1960-1970 représentèrent l'âge d'or de la citoyenneté industrielle au Canada, avec la syndicalisation des travailleurs du secteur public, les progrès de la lutte contre la discrimination, ainsi qu'une vague de réformes en matière de normes du travail et de santé-sécurité au travail. À partir des années 1980 toutefois, l'émergence du néo-libéralisme, l'accord de libre-échange avec les États-Unis et le gel des salaires dans le secteur public se traduisent par un retour en force des contraintes liées au marché, exacerbées au demeurant par la dynamique de la mondialisation, le déclin du taux de syndicalisation, la détérioration des conditions de travail, la précarisation de l'emploi, tout cela sur fond de crise de l'État social. Fudge identifie deux cas de figure qui pourraient succéder à celle de la citoyenneté industrielle : soit la citoyenneté marchande, où règnent l'individualisme, la porosité du filet de protection sociale et la recontractualisation du travail ; soit la citoyenneté au travail, qui ne se limite plus au domaine de l'emploi mais couvre toutes les formes du travail. Ces deux types ne font qu'illustrer deux visions possibles et opposées de ce qu'il peut advenir de notre régime de citoyenneté. Ce qui est certain, c'est que toute conception future de la citoyenneté doit répondre aux défis de la mondialisation, du néo-libéralisme et de la féminisation de la main-d'œuvre.

La contribution de Peter Berg et Ann Frost s'intéresse à la « dignité au travail » des travailleurs occupant des emplois faiblement rémunérés et peu qualifiés dans le secteur des services. La dignité au travail implique ici un traitement équitable au travail, la sécurité économique et un travail intrinsèquement satisfaisant. Sur la base d'une étude des travailleurs dans les hôpitaux aux États-Unis, les auteurs mesurent l'effet des caractéristiques du milieu de travail sur la dignité au travail, tel que le ressentent les travailleurs interrogés. Rejoignant la littérature classique sur la qualité des emplois, les auteurs supposent qu'un travail monotone, routinier et non stimulant intellectuellement ne peut être considéré comme satisfaisant ; que la sécurité économique se mesure suivant le taux de la rémunération; et 
qu'un traitement inéquitable apparaît lorsque l'attitude de l'employeur à leur égard est irrespectueuse, capricieuse et arbitraire. Plus précisément, Berg et Frost trouvent que si les changements dans l'organisation du travail, tels que présentés dans la littérature sur les « milieux de travail performants » n'ont pas d'impact sur la perception de la dignité au travail, c'est tout le contraire pour ce qui est de l'effet de la formation professionnelle. De manière générale, la présence syndicale n'influe pas directement sur le sentiment de dignité, sauf quant à la rémunération, qui est justement plus élevée lorsque l'implication syndicale est forte. La disponibilité de ressources adéquates pour effectuer le travail a aussi un impact positif - ceci correspond au constat de Hodson (2001) quant aux facteurs renforçant la citoyenneté organisationnelle -, alors que c'est l'inverse lorsque les travailleurs ressentent une surcharge dans leur travail. Les auteurs arrivent ainsi à identifier un ensemble de conditions minimales d'un travail décent, qui pourrait assurer une certaine citoyenneté au travail pour ces salariés moins rémunérés et peu qualifiés.

Le texte de Marie-Josée Legault porte sur la «crise » du mouvement syndical qu'elle analyse sous un angle identitaire, celui de la segmentation du collectif syndical due à la diversification croissante de la main-d'œuvre syndiquée. Les chartes de droits de la personne apportent un appui juridique capital à ce mouvement de diversification des intérêts et des valeurs, par la reconnaissance qu'elles font des droits des minorités dans les milieux de travail : par exemple, les membres des groupes cibles (femmes, minorités ethniques) visés par les programmes d'accès à l'égalité (PAE) ou les jeunes, victimes de la présence de clauses de disparité de traitement (dites « clauses orphelins ») créant des disparités intergénérationnelles. Comme le remarque l'auteure avec acuité, la notion d'égalité véhiculée par les chartes des droits entre en conflit avec le concept équivalent utilisé par les organisations syndicales et qui postule une similitude d'intérêts de classe des membres d'une unité de négociation. Les revendications des groupes minoritaires au sein du syndicat sont sources de conflits et remettent en cause la conception «majoritaire» de la démocratie syndicale. Qu'il s'agisse des PAE, du harcèlement sexuel ou des mesures de conciliation travail-famille, ces politiques provoquent des heurts au sein de la main-d'œuvre, dans la mesure où certains y voient un traitement de faveur à l'endroit des travailleuses. L'auteure signale au passage la problématique distincte des clauses de disparité de traitement, laquelle a vu la constitution de collectifs de défense des droits des jeunes travailleurs en dehors des instances syndicales. Cette segmentation du collectif au travail exerce forcément une influence sur l'acteur syndical. Au modèle de la « citoyenneté industrielle » fondé sur la seule structure syndicale officielle est opposée la revendication d'une nouvelle « citoyenneté syndicale » de la part des travailleurs appartenant à des 
minorités. Certes, des conflits d'intérêts (et de valeurs) ont toujours traversé les rangs des organisations syndicales : ce qui est nouveau, toutefois, c'est l'apparition (sous l'égide des chartes) d'arènes juridiques où ces conflits pourront être tranchés, en dehors du domaine traditionnel du droit du travail. Seul l'avenir permettra de voir si les organisations et représentants syndicaux arriveront à gérer ces nouveaux conflits, tout en préservant leur capacité d'action face à l'employeur.

L'article de David Peetz traite du problème des «resquilleurs » ou des «profiteurs » (free riders) dans le domaine des relations du travail en Australie. David Peetz établit d'abord que la citoyenneté industrielle prescrit à la fois des droits et des responsabilités : notamment le droit de bénéficier de la négociation collective, mais aussi l'exigence d'un comportement responsable vis-à-vis des autres membres du collectif syndical. Se pose ainsi toute la question éminemment délicate des dynamiques sociales autour du collectivisme et de l'individualisme. Il met en doute la position d'un auteur comme Putnam (2000), lequel croit qu'on assiste à un déclin généralisé du capital social dans plusieurs pays occidentaux. Le phénomène croissant du free riding en Australie s'explique par d'autres facteurs, selon Peetz, en particulier par un programme législatif néo-libéral qui affaiblit la position des syndicats en Australie et rend illégale la syndicalisation obligatoire ou le paiement d'une compensation par les non-adhérents aux syndicats. Non seulement cette situation provoque un problème d'équité quant aux droits et responsabilités des citoyens au travail, mais elle fausse l'arbitrage qui doit jouer entre l'individu et le collectif au regard de la régulation du travail. Peetz compare l'Australie avec la situation canadienne qui connaît la «formule Rand» ou retenue obligatoire des cotisations syndicales. L'auteur estime que des changements législatifs, tout comme une modification des pratiques syndicales, sont nécessaires pour endiguer le free riding en Australie.

Arnulfo Arteaga Garcia plaide en faveur de l'émergence d'une nouvelle citoyenneté, devant l'impact de la mondialisation sur l'économie mexicaine. Les transformations économiques en cours ont un impact profond non seulement sur les conditions de travail, mais sur les conditions de vie des travailleurs mexicains. L'étude distingue quatre grands secteurs dans l'économie mexicaine. Si le secteur manufacturier se caractérise par l'introduction de nouvelles formes d'organisation du travail et de systèmes de travail à haute performance, ces nouvelles pratiques managériales ne se traduisent pas, bien au contraire, par une amélioration de la condition des travailleurs et une participation accrue aux décisions économiques de leurs entreprises. La croissance du secteur informel — très présent dans toutes les économies en développement - se fait par définition en dehors du cadre légal et participe souvent d'activités illégales : il ne saurait être question ici 
d'une quelconque citoyenneté au travail. Cette déformalisation du travail au Mexique trouve son prolongement dans la situation des travailleurs migrants aux États-Unis, lesquels constituent un cinquième de la force de travail mexicaine. Il s'agit ici d'une formidable source de devises étrangères pour l'économie mexicaine ; quant aux travailleurs eux-mêmes, il sont placés dans une situation d'illégalité, les entrepreneurs américains profitant d'un apport considérable de main-d'œuvre faiblement rémunérée. Enfin, l'émergence des emplois dans le secteur des nouvelles technologies de l'information et des communications ne doit pas être rattaché trop rapidement au paradigme d'une nouvelle «économie du savoir» car la plupart de ces emplois se caractérisent par de bas salaires et un travail routinier, sans le bénéfice, vu la précarité, des normes juridiques encadrant d'autres formes de travail. En conclusion, l'auteur souligne que la mondialisation promeut l'émergence du travail flexible et polyvalent, dans un contexte d'incertitude lié au caractère aléatoire de l'économie générant des formes croissantes d'exclusion de la protection du travail. Le démantèlement de l'État social au Mexique remet par ailleurs en question le schéma évolutionniste classique de la citoyenneté au travail que l'on trouve chez T.H. Marshall. La solution, face aux dérives de la mondialisation, réside dans la construction d'une citoyenneté globale, qui ne peut être que la résultante de l'activité des acteurs sociaux cherchant à réassurer la position centrale du travail dans l'élargissement des droits des citoyens et assurant un lien entre les revendications des travailleurs au Sud comme au Nord.

Yves Hallée aborde la question de la citoyenneté au travail sous l'angle identitaire. Il s'intéresse au rôle des valeurs dominantes dans la construction des identités sociales, en l'occurrence celles qui sous-tendent la reconnaissance du travail des femmes. La citoyenneté s'est construite historiquement en référence aux rôles masculins, les femmes étant tout simplement exclues de la citoyenneté. Dans la réflexion contemporaine, on peut différencier trois compréhensions différentes de la citoyenneté visant la place des femmes dans la société : une citoyenneté neutre par rapport au genre, centrée sur l'égalité des droits et des obligations entre les hommes et les femmes, qui oblitère l'existence historique de rapports de domination entre les hommes et les femmes et l'insuffisance des mesures d'égalité des chances; une citoyenneté déterminée par le genre, centrée sur la reconnaissance des valeurs traditionnellement associées aux rôles féminins : «l'éthique de la sollicitude », la compassion et le travail centré sur autrui (le care); enfin, une citoyenneté pluraliste, qui ne fait du genre qu'un vecteur parmi d'autres (âge, statut social, état de santé, sexualité, etc.) sous-tendant la construction des identités sociales. Dans cette dernière perspective et prenant comme point de référence la question de l'équité salariale, Yves Hallée estime que la discrimination salariale subie par les femmes découle historiquement de 
la moindre valeur accordé au travail domestique et aux activités de soins. Il plaide en faveur d'une féminisation du travail des hommes pour que progresse l'équité salariale, la dissémination des valeurs de sollicitude et de compassion associées au travail féminin devant se traduire par une pleine reconnaissance de la valeur économique et sociale de ce travail.

Enfin, pour Georgina Murray, l'utilité de la notion de citoyenneté au travail est de fournir des principes directeurs en matière de politiques publiques ou privées. Ceci dépend de la légitimité que peut acquérir cette notion, de manière à s'imposer dans le discours public et chez les décideurs. Le problème demeure toutefois qu'une véritable citoyenneté au travail est fortement limitée dans le cadre d'une économie capitaliste orientée par les politiques libérales de la classe dominante. Georgina Murray brosse à cet égard un tableau des principaux courants identifiés à l'économie classique : certes, ces courants n'ont pas produit le capitalisme, mais ils ont grandement contribué à sa légitimation. Qu'il s'agisse des théories libérales initiales ou de leur radicalisation par le néo-libéralisme, toutes ces conceptions partagent l'idée d'une réduction des coûts de la main-d'œuvre et d'accroissement de la productivité. À ces théoriciens du libéralisme économique il faut opposer, d'une part, les tenants du Welfare State, à commencer par Keynes ; d'autre part, les partisans du marxisme, qui ne croient pas qu'un retour à la démocratie sociale soit viable dans les conditions actuelles de l'économie capitaliste. Le libéralisme économique, foncièrement inégalitaire, entre également en conflit avec diverses conceptions de la citoyenneté au travail. Pour Georgina Murray, il importe de dépasser le cadre national, lequel n'est pas en mesure de contrer efficacement le libéralisme à l'heure de la mondialisation. Il faut désormais penser une citoyenneté globale, dont les porteurs spécialisés seront les mouvements de travailleurs et qui visera à garantir une gamme étendue de droits économiques pour l'ensemble des citoyens. La notion de citoyenneté au travail y prendrait alors tout son sens.

Ce bref survol des contributions réunies dans ce numéro spécial sur la citoyenneté au travail laisse évidemment de côté certaines des dimensions abordées. Cela dit, nous croyons important de relever un certain nombre de thèmes communs.

En premier lieu - abstraction faite de la tentation téméraire de ceux qui étudient par profession le travail et l'emploi d'une recherche à tout prix de la cohérence - il y a nettement un effort rigoureux de parvenir à une vision plus articulée et plus englobante des choses. Le paradigme de la citoyenneté au travail est potentiellement porteur d'une telle cohérence, encore que ses significations multiples et le pluralisme épistémologique qui 
le fonde invitent très certainement à la prudence et à une exigence accrue de rigueur.

En deuxième lieu, une bonne part de cet effort de recherche trouve sa source dans l'écart croissant entre les modèles traditionnels de la sphère du travail et de sa régulation par les politiques publiques institutionnalisées, et les transformations en cours dans la vie concrète du travail. Et ce, d'autant plus qu'un grand nombre d'emplois semblent se détériorer, caractérisés par une insécurité chronique et par une aliénation accrue par rapport aux résultats du travail (voir, par exemple, Green, 2006), pendant que d'autres salariés au contraire voient constamment leur situation progresser, ce qui se traduit dans certains milieux de travail par l'atteinte d'une citoyenneté organisationnelle d'un niveau supérieur. Face à une telle disparité, il y a une sorte de fracture des cadres institutionnels nationaux, incapables d'agir efficacement dans l'un ou l'autre cas.

En troisième lieu, au-delà des caractéristiques spécifiques de l'emploi, la nécessité très claire d'un récit englobant ne fait pas de doute : il s'agit ici de rendre compte également des caractéristiques des personnes susceptibles d'occuper les emplois concernés, en gardant à l'esprit que «l'emploi » a vocation à inclure non seulement les tâches de pointe dans les entreprisesclés sur le plan international, mais tout aussi bien le travail lié au «care » et à la reproduction sociale. Ceci implique de développer une articulation nouvelle entre formes différentes d'identités : individuelles et collectives, appartenance au Nord et au Sud, productives et reproductives, féminines et masculines en tenant compte des différentes phases du cycle de vie, pour n'en nommer que quelques unes. Ceci soulève au surplus le jeu complexe qui se tisse entre l'individualisme et le collectivisme, entre l'individu et le groupe et aussi entre le statut et l'identité, tout autant qu'il nous impose de repenser les instruments juridiques et politiques visant à conférer un statut et à déterminer une identité.

En quatrième lieu, on ne peut qu'être frappé du fossé énorme qui existe entre l'aspiration à une citoyenneté transnationale telle que véhiculée par le discours transcendant sur le travail décent (Commission mondiale sur la dimension sociale de la mondialisation, 2004), et, tel que l'illustre avec tant d'éloquence le texte d'Arteaga dans le présent numéro, la situation paradoxale des travailleurs migrants illégaux d'origine mexicaine aux États-Unis, lesquels doivent travailler en-dessous des normes minimales du travail et sont en même temps incapables d'avoir accès à un minimum de droits tant dans leur contrée d'origine que dans leur pays d'adoption. Ce fossé entre aspirations et réalités va continuer à agir puissamment dans le sens d'une réflexion sur les possibilités, mais aussi sur les écueils de la citoyenneté au travail, de même que sur son potentiel de transformation (voir aussi Johnston, 2000). 
En cinquième lieu, il existe un besoin évident de synthèses en matière de politiques publiques, visant à construire des ponts entre ces écarts, tensions et fragmentations. Tous nos contributeurs semblent d'accord sur le fait qu'une foi aveugle dans les lois du marché ne peut, en toute probabilité, conduire de manière adéquate vers une réelle citoyenneté au travail. Tous en appellent au contraire à la (re)construction d'une forme de cohérence en matière de politiques publiques. En même temps, personne ne suggère qu'il soit possible de revenir vers les points de départ du passé, qu'il s'agisse d'une reconstitution nostalgique, tels qu'ils existaient à l'origine, du droit collectif du travail ou de l'État-providence, du fait notamment des changements significatifs dans l'organisation du travail et de leur rapports aux dynamiques socio-économiques globales.

En sixième lieu, l'une des conclusions les plus stimulantes qui émane de ces contributions concerne le caractère négocié de la citoyenneté au travail. On retrouve ici une intuition centrale, celle d'une vision du travail comme processus continu de régulation. La mondialisation fournit ainsi autant d'occasions pour (re)réguler à la fois le travail et le procès même de régulation (Murray, Lévesque et Vallée, 2000). Voilà qui exige d'étudier ces processus en cours suivant des approches variées, et met aussi en lumière l'importance d'une interaction entre les acteurs sociaux, traditionnels et émergents. En somme, la citoyenneté fournit une voie analytique pour une (re)régulation complexe du travail : on doit l'entendre à la fois comme un «work in progress » et comme un discours aspirationnel pour les acteurs sociaux à la recherche d'une transformation des milieux de travail.

La véritable «bourse conceptuelle » qu'ouvre ce numéro de la revue $R I / I R$ vise à stimuler des perspectives innovatrices et des contributions scientifiques futures. Notre introduction ne fait que gratter un peu la surface du riche contenu des textes qui suivent. Il reviendra à nos lecteurs, nous l'espérons, de s'approprier la réflexion stimulante qui anime ces textes. Quant à nous, nous espérons seulement remettre à jour certains des défis analytiques que Harry Arthurs a brillamment identifiés en 1967, en invitant la communauté scientifique à penser de manière plus globale la problématique du travail et ce, en insérant les conditions de vie et d'emploi des travailleurs dans la catégorie fondamentale de la citoyenneté.

\section{BIBLIOGRAPHIE}

ARTHuRs, Harry. 1967. « Developing Industrial Citizenship : A Challenge for Canada's Second Century ». Canadian Bar Review, XLV, 786-830.

ARTHURS, Harry. 1996. « Labour Law without the State ». University of Toronto Law Journal, 46, 1-45.

ARTHURs, Harry. 1999. « The New Economy and the New Legality : Industrial Citizenship and the Future of Labour Arbitration ». Canadian Labour and Employment Law Journal, 7 (1), 46-63. 
Castells, Manuel. 1996. The Rise of the Network Society. Oxford : Blackwell Publishers.

COMMISSION MONDIALE SUR LA DIMENSION SOCIALE DE LA MONDIALISATION. 2004. Une mondialisation juste : créer des opportunités pour tous. Genève : Organisation internationale du travail.

Coutu, Michel. 2004. «Industrial Citizenship, Human Rights and the Transformation of Labour Law : A Critical Assessment of Harry Arthurs' Legalization Thesis ». Revue canadienne Droit et Société/Canadian Journal of Law and Society, 19, 73-92.

FRANCFORT, Isabelle, Florence OSTY, Renaud SAINSAULIEU et Marc UhALDE. 1995. Les mondes sociaux de l'entreprise. Paris : Deslée de Brouwer.

Fudge, Judy et Leah Vosko. 2001. «By Whose Standards? Reregulating the Canadian Labour Market ». Economic and Industrial Democracy, 22, 327-356.

GreEn, Francis. 2006. Demanding Work: The Paradox of Job Quality in the Affluent Economy. Princeton : Princeton University Press.

HABERMAS, Jürgen. 1996. "Citizenship and National Identity ». Between Facts and Norms. Contributions to a Discourse Theory of Law and Democracy. Trad. de William Regh. Cambridge : MIT Press, 491-515.

HABERMAS, Jürgen. 2000. Après l'État-nation : une nouvelle constellation politique. Trad. de Rainer Rochlitz. Paris : Fayard.

HASSENTEUFEL, Patrick. 1996. «L'État-providence ou les métamorphoses de la citoyenneté ». L'Année sociologique, 46 (1), 127-149.

Held, David. 1995. Democracy and the Global Order. Cambridge : Polity Press.

Hodson, Randy. 2001. Dignity at Work. Cambridge : Cambridge University Press.

Johnston, Paul. 2000. «The Resurgence of Labor as Citizenship Movement in the New Labor Relations Environment ». Critical Sociology, 26 (1-2), 139-160.

Kahn-Freund, Otto. 1954. «Legal Regulation». The System of Labour Relations in Great Britain. A. Flanders et H. Clegg, dir. Oxford : Oxford University Press, 42-127.

Kerr, Clark, John Dunlop, Frederick Harbison et Charles Myers. 1960. Industrialism and Industrial Man. Cambridge : Harvard University Press.

Marshall, T. H. 1949. "Citizenship and Social Class ». Réimpression dans Sociology at the Crossroads and other Essays, 1963. London: Heinemann, 67-127.

Murray, Gregor, Christian LÉvesque et Guylaine ValléE. 2000. « The Reregulation of Labour in a Global Context: Conceptual Vignettes from Canada ». The Journal of Industrial Relations, 42 (2), 234-257.

MurRAY, Gregor, Jacques BÉlAnger, Anthony Giles et Paul-André LAPOINTE, dir. 2004. L'organisation de la production et du travail : vers un nouveau modèle? Québec : Presses de l'Université Laval. 
Piore, Michael. 1995. Beyond Individualism: How Social Demands of the New Identity Groups Challenge American and Political Life. Cambridge : Harvard University Press.

Putnam, Robert D. 2000. Bowling Alone: The Collapse and Revival of American Community. New York : Simon and Schuster.

SChnAPPer, Dominique. 1994. La communauté des citoyens. Paris : Gallimard.

SCHNAPPER, Dominique. 1997. Contre la fin du travail. Paris : Éditions Textuel.

SCHNAPPER, Dominique. 2002. La démocratie providentielle : essai sur l'égalité contemporaine. Paris : Gallimard.

Stasiulis, Davia K. et Abigail B. BaKan. 2005. Negotiating Citizenship : Migrant Women in Canada and the Global System. Toronto : University of Toronto Press.

Stone, Katherine W. 2004. From Widgets to Digits : Employment Regulation for the Changing Workplace. Cambridge : Cambridge University Press.

Vallée, Guylaine, Michel Coutu et Marie-Claude Hébert. 2002. «Implementing Equality Rights in the Workplace : An Empirical Study ». Canadian Labour and Employment Law Journal, 9, 71-117. 


\title{
Towards Citizenship at Work?
}

\section{An Introduction}

\author{
Michel Coutu \\ Gregor MurRay
}

Harry Arthurs (1967) expressed much of the optimism for labour's long march to full civil, political and social rights in modern industrial societies in his 1960s path-breaking article "Developing Industrial Citizenship: A Challenge for Canada's Second Century". His reading offers a compelling narrative of the evolution of labour law in Canada, both past and future, as the gradual and necessary affirmation of industrial citizenship. T.H. Marshall (1949) had already traced the development of the different dimensions of citizenship rights in a highly influential reading of two centuries of industrial progress achieved by British workers. Although such a citizenship was not yet fully achieved, its main traits were increasingly evident in the development of both the postwar welfare state and in the increasing maturity of an autonomous body of collective labour law (see, for example, Kahn-Freund, 1954). Arthurs built on this vision and undoubtedly echoed some of the pervasive optimism of the period in foreseeing a seemingly inevitable and necessary affirmation of collective rights in the economic sphere, which he labelled "industrial citizenship". Just as Kerr, Dunlop, Harbison and Myers (1960) viewed the maturation of industrial societies towards stable forms of industrial pluralism in the world of work, in which legitimately contending collective rights would come to form agreed norms on the basis of recognized collective rights, so Arthurs anticipated a future trajectory for Canadian public policy on work and collective labour law.

This vision was predicated on the crystallisation of these collective labour rights. Underpinned by the pluralism of collective actors at the (industrial) workplace, it was an integral part of a broader framework of economic and social citizenship towards which societies should and would evolve. Thus this traditional notion of industrial citizenship embraced a view

- Coutu, M., and G. Murray, School of Industrial Relations, Université de Montréal and Interuniversity Research Centre on Globalization and Work (CRIMT - Université de Montréal, Université Laval, HEC Montréal). 
of the progressive extension of the realm of freedom for workers: first, in the form of individual political rights, gradually entailing universal suffrage by the early twentieth century; then, as in the establishment of collective labour rights in contrast to the primacy of property rights, such as the positive right to associate for the purposes of negotiating terms and conditions of work and in the promotion of collective associations for workers to face the corporations that they worked for; and, finally, to social rights associated with the implantation of the welfare state in the post-World-War- II period, such as economic and social security for all including access to healthcare and education. In the field of industrial relations, both collective bargaining and the provision of collective and individual rights by the state as a means for ensuring the true exercise of this industrial citizenship were bound to grow.

In retrospect, one cannot but be struck by the way that this roadmap to the future, encapsulated as industrial citizenship, constituted a paradigm for the fields of industrial relations, labour law and public policies about work. Among the core premises which rallied consent, that labour is not a commodity, that it is invested of human agency and that such humanity, sometimes in opposition to the operation of the market, must be recognized in the ways that legitimately contending social actors create norms, procedures and rules about work. Not only has the idea of industrial citizenship served as a normative template for judging the success of work relations in industrial systems but it has increasingly made its way into international instruments defining fundamental human rights. Arthur's article is therefore an important intellectual landmark of where we once thought we might go, and readers would do well to savour it anew, but most observers, including Arthurs himself (1996 and 1999), would now readily agree that this is not exactly where we went.

The workplace in Canada and elsewhere has changed significantly over the past decades and the pace of change appears to be ever accelerating. New information and communications technologies (Castells, 1996), the increasing internationalization of the production of goods and services (Held, 1995), a revolution in the management of production and work organization (Murray et al., 2002), the dis- and relocation of workplaces across both time and space (Stone, 2004) and the multiplication and fragmentation of individual and collective social identities in and beyond the workplace (Francfort et al., 1995, Piore, 1995) are just some of the factors driving these changes. The extent of change, moreover, leads to an increasing "disconnect" between, on the one hand, the prevailing institutional framework for work and employment and the sets of assumptions that informed it, and, on the other hand, these current changes sweeping the world of work. 
The notion of industrial citizenship was rooted in an older model of production and predicated on assumptions increasingly open to question. The workers concerned by the notion of collective labour rights tended to be in a predominantly male realm of formal labour market activity, according to a model of full-time, permanent employment, as opposed to an overwhelmingly female realm of unpaid domestic labour concerned with caring and social reproduction (Fudge and Vosko, 2001). Given this bias towards manufacturing and longer job tenure with the same employer, it was also assumed that the bulk of employees would be represented collectively by trade unions. Yet, changes in the distribution of employment, the organization of work into more fragmented units, and the relative decline of collective representation raise important questions about both job quality and collective representation about work. At the very least it is no longer sufficient to speak of industrial citizenship because work is largely beyond the confines of "industry". More tellingly again, these changes suggest a rethinking of the boundaries of work, raising gender issues about work beyond the formal labour market.

Similarly, the growing assertion of other individual and collective identities (gender, ethnicity, sexuality, "professionality") has led to a much stronger emphasis on the codification of other rights, especially equality rights, with important consequences for collective labour law. While collective labour law once offered an encompassing and even self-sufficient paradigm for the understanding of citizenship at work, the affirmation of new identities and the multiplication of instances to ensure their expression, application and interpretation have challenged this traditional paradigm. The competing institutional interpretations of such citizenship are sometimes complementary to the traditional expression of collective labour rights, but also sometimes in conflict (Vallée, Coutu and Hébert, 2002).

Finally, the redefinition of the role of the state in many industrialized economies has also challenged prevailing notions of economic and social security, opposing notions of individual responsibility to those of collective entitlement. The institutional arrangements that underlay the citizenship of collective entitlement expressed past trade-offs between the organized working class and the state that no longer apply in the same way. And such rights were based on modes of governance in national territories rather than on transversal notions of human rights and global citizenship.

This affirmation of a wider range of individual and collective social and professional identities, the implantation of new organizational models for the production of goods and services and the increasing transnationalization of economic and social relations therefore open up rich avenues of enquiry as regards how we might appropriately define citizenship at work in a global era. 
Such is the point of departure for this special issue of Relations industrielles/Industrial Relations. Drawing on a range of original contributions, it seeks to set out some of the pathways for thinking about citizenship at work. Under the auspices of its Social Sciences and Humanities Research Council's Major Collaborative Research Initiatives (SSHRC-MCRI) project on rethinking institutions for work and employment in a global era, the Inter-University Research Centre on Globalization and Work (CRIMT - Université de Montréal, Université Laval, HEC Montréal), in cooperation with a range of labour market and public policy partners, issued a call for papers to be presented at it June 2004 international colloquium on Citizenship at Work: Thinking the Workplace of the Future. ${ }^{1}$ Organized by CRIMT and held at Université Laval, this colloquium featured a range of original papers and contributions, stemming from a selection of both the responses to our call for papers and invited contributions from leading academic specialists and labour market partners. The articles in this special issue of $R I / I R$ are those that were subsequently reviewed by external referees. ${ }^{2}$ In the remainder of this introduction, we set out and assess some of the pathways that emerge from this diverse and rich collection of studies.

$$
* * *
$$

Although the concept of citizenship can be powerfully resonant in its articulations to both democracy and the State, it is also a vessel that carries many meanings (Schnapper, 1997a: 73)—all the more so when applied to the realm of work. Multiple derivations are at play: industrial citizenship, social citizenship, organizational and firm citizenship and global citizenship, to name just a few. At the very least, some attempt to achieve greater conceptual clarity would seem to be in order.

The concept of citizenship can be descriptive; it can also be normative or prescriptive. Sometimes, it is used in both ways, even without distinction.

1. Financial, organizational and material support for this colloquium were provided by the Social Sciences and Humanities Research Council of Canada, the Fonds québécois de recherche sur la société et la culture, Human Resources and Skills Development Canada's Labour-Management Partnerships Program, Développement économique et régional de recherche, Ministère du travail, International Labour Office, the Canadian Labour and Business Centre, the Canadian Policy Research Networks, Relations industrielles/ Industrial Relations and Université Laval and its Industrial Relations Department. We particularly wish to thank Judith Paquet and Nicolas Roby for their work on the academic organizing committee as well as Christian Brunelle, Jean Charest and Pierre Verge for their role in the selection of the call for papers and the anonymous reviewers for this special issue.

2. A second stream of invited papers, surveying key themes and dimensions of citizenship, will soon be published in book form: Travail et citoyenneté: Quel avenir?; Broken Paradigms: Rethinking Citizenship at Work in a Global Era. 
When the idea of citizenship expresses a project or an ideal or even a utopian aspiration, as with Habermas's $(1996,2000)$ supranational citizenship, then its use is normative. When used to give an empirical take on the current state of affairs, as with both T.H. Marshall (1949) and Schnapper (1997, 2002), then it is descriptive. Even if values inevitably inform the way that this current state of affairs might be interpreted, the descriptive approach is focused on a sociological account of the evolution of citizenship-how things are-as opposed to a philosophical or moral interpretation-as in how things should be.

Another way of conceptualizing citizenship opposes identity to status. Status refers to the socio-legal status of a group of individuals as regards, for example, their fundamental rights (civil, political, social, cultural, etc.). In contrast, identity concerns the sense of belonging, as manifested by an individual relative to some larger entity, be it the national state or a religious, ethnic, linguistic or cultural community. The work of T.H. Marshall is largely concerned with citizenship status, inasmuch as the focus is on the basic rights achieved historically by English citizens. Schnapper, on the other hand, is primarily concerned with citizenship identity because she focuses on the social relations between members of a community of citizens as the source of a sense of political citizenship.

Locating discussions of citizenship along both of these axes provides some degree of conceptual clarity as it is possible to situate authors in any one of the four cells of the matrix. Habermas, for example, is easily located in terms of both the normative and identity dimensions of citizenship whereas T.H. Marshall's work is both descriptive and concerned with status (Hassenteufel, 1996).

The objective is certainly not to promote one or another of these possible conceptual tandems but rather to invite greater clarity in the way that we think about citizenship at work. Each of these dimensions is useful but it is necessary to think about the different kinds of analysis that they entail.

There is, moreover, yet another degree of complexity in this discussion, and herein resides at least some of the richness to be found in the contributions to this special issue. One of the reasons that citizenship offers so many meanings is because the concept is itself in movement- the subject of continuous negotiation among social actors. The evolutionary take offered by T.H. Marshall highlights the gradual acquisition of basic rights and how the formal equality of civil rights conflicted with socio-economic inequalities of social class, hence the growing importance of new social rights and the search for more significant forms of equality. Similarly, while Arthurs' methodological posture in his 1967 article was largely descriptive 
and explanatory, it also expressed an ideal—a vision for the future-which, although it was not be achieved in the short or medium term for the reasons explored above, remains a powerful statement of what could be (Coutu, 2004). Indeed, we think that it is precisely this tension between what is and what should be that provides a roadmap for the evolution of public policies on work.

The same can be said of the tension between status and identity. We emphasized above the importance of the proliferation of identities related to working lives. The question to some degree is whether status is conferred on these identities? This is exactly the line of analysis at the core of much of the research on equality in an era of charter rights. In many ways, the current period is an ongoing experiment in the negotiation between status and identity. Stasiulis and Bakan (2005), for example, offer a fascinating study of citizenship on the part of migrant women workers in Canada. This is a case of a variety of identities in search of status - those of women, of persons of colour, of workers, of nationality - but the recognition of such identities must be mobilized by social actors. In this sense, it is a continuous process, still far from being achieved and indeterminate in terms of final results, but yet a project informed by the understandings of these persons in their multiple identities.

The interest in discussions about citizenship at work flows then from this understanding of the tensions between what is and what should be, and between the affirmation of identities and their lack of recognition in terms of status. That the world of work is undergoing a tremendous period of change and that we can observe huge gaps between what is and what could or should be is a veritable convocation to think about the negotiation of citizenship at work by both people at work and those excluded from it. Such negotiations concern both of the axes of citizenship explored above: identity as well as status; in both descriptive and normative dimensions. This is certainly the vocation of one of the cross-cutting themes in CRIMT's own research program; it is also the motive behind the organization of an International Colloquium on Citizenship at Work and this special issue of $R I / I R$. Let us now turn to the detailed contents of this special issue.

Judy Fudge's article offers a scintillating overview of the evolution of citizenship at work in Canada. She perceives the erosion of the notion of "industrial citizenship", at least as it was understood in the post-WorldWar-II period. In referring to the work of Harry Arthurs (1967), Fudge defines industrial citizenship as a status limiting the commodification of labour. This status is based on a range of both individual and collective 
rights and it is inextricably linked to the development of an interventionist welfare state. Through an historical overview of this industrial citizenship regime in Canada, Fudge emphasizes its limited scope (particularly for women) and its weak institutionalization. The origins of this regime can be dated to the affirmation of the right to unionize and the institutionalization of collective bargaining. It should, however, be kept in mind that this was a male breadwinner model, indifferent-and so too were unions at the time - to the rights of women. The 1960s and 1970s were in many ways the golden age of industrial citizenship in Canada. The unionization of public sector workers, progress in the struggles against discrimination at work and multiple reforms in minimum standards and health and safety at work were just a few of the accomplishments. From the 1980s onwards, however, with the rise of neo-liberalism, free trade and public sector financial constraints, we witness a return to market constraint, exacerbated even further by the dynamics of globalization, a decline in levels of unionization, deteriorating working conditions and a rise in precarious work, all of which have as backdrop a crisis of the Welfare State. Fudge then identifies two scenarios for industrial citizenship in the future: market citizenship, characterized by rampant individualism, the permeability of social safety nets and the commercialization of work; and citizenship at work, which is not just limited to employment but would include all forms of work. These scenarios are meant to illustrate possible but opposing visions of the future evolution of our industrial citizenship regime. What is essential in Fudge's view is that any future conception of citizenship as it relates to work must be able to respond to the triple challenge of globalization, neo-liberalism and feminization of the workforce.

The contribution of Peter Berg and Ann Frost focuses on "dignity at work", as it relates to low wage and low skill service sector workers. Dignity at work here refers to equitable treatment in the workplace, economic security and a job that is intrinsically satisfying. On the basis of a study of hospital workers in the United States, the authors assess the effects of workplace characteristics on dignity at work, as experienced by these low wage and low skill healthcare workers. Drawing on the classic contributions on job quality, Berg and Frost hypothesize as regards conditions leading to low levels of dignity at work. These include: monotonous and intellectually under stimulating work; economic insecurity, as measured by low pay; and inequitable treatment, in terms of disrespectful and arbitrary behaviour on the part of managers. In fact, Berg and Frost find that changes in work organization, at least as envisaged in the high-performance work organization literature, appear to have little impact on the experience of dignity at work; rather, it is the presence or absence of training that seems to make a difference. Unionization per se is also not a predictor of dignity; though the 
higher levels of pay associated with a stronger union role in a workplace do exert a positive impact on the experience of dignity at work. Consistent with Hodson's (2001) findings on what reinforces organizational citizenship, adequate resources to accomplish the job also have a positive impact, whereas excessive workload exerts the opposite effect. In identifying a set of minimum conditions associated with what might be labelled decent work, the authors are thus able to make a key contribution in terms of developing citizenship at work for the low paid and low skilled.

Marie-Josée Legault's article uses the lens of identity politics to consider the new challenges for the union movement. Her particular concern relates to the segmentation of collective union identities in the context of the growing diversity of the unionized workforce. Through their specific recognition of the rights of minorities in the workplace, charters of human rights certainly reinforce the continuing diversification of interests and values. These include targeted groups (women and visible minorities) coming under the ambit of equal access programs and young workers who are victims of intergenerational disparities because of the implementation of two-tier systems of wages and benefits. Legault explores with acuity how notions of equality, as expressed in charters of rights, conflict with equivalent notions used by union organizations that posit a similarity of interest of all members in a bargaining unit. Claims made by minority groups within a union are thus a source of conflict because they often question the prevailing majority rule that is at the core of union democracy. Be it in terms of equal access programs, sexual harassment or work-family balance, these policies can engender tensions within the workforce because they are seen by some to "favour" particular interests, notably those of women workers. To illustrate yet further these tensions, Legault also explores how two-tier wage systems have led to the creation of autonomous groupings dedicated to the defence of young workers. These groupings operate fully outside of formal mechanisms of union governance. This segmentation of collective identities certainly influences unions. In the place of a model of industrial citizenship centred on the union, as officially constituted, we see emerging claims for a new "union citizenship" on the part of workers belonging to these identity groups. Of course, writes Legault, conflicts of interests (and values) have always cut across union ranks. What is new in the era of charters of rights, however, is the emergence of judicial arenas, beyond the scope of traditional labour law, where such conflicts can be arbitrated. Only the future will tell how union organizations and their representatives succeed in managing these conflicts, at the same time as they preserve their capacity for action vis-à-vis employers.

David Peetz's contribution considers the free rider problem in the context of Australian labour relations. For Peetz, industrial citizenship 
entails both rights and responsibilities: notably, the right to benefit from collective representation; but also responsible behaviour with regard to other members of a group of workers. Herein lies the thorny question at the heart of Peetz's study as to how to achieve balance between collectivism and individualism. Peetz doubts the plausibility of the Putnam (2000) hypothesis as regards a secular decline of social capital in western nations. Instead, he believes that the rise of a free rider problem in Australia is attributable to a neo-liberal legislative agenda which has prohibited both compulsory unionism and the payment for union services by non-union members. In his view, not only does this situation lead to injustices in the balance between the rights and the responsibilities of citizens at work, but it skews the kind of trade-offs that should be made between individuals and the collectivity in the regulation of their work. When the Australian case is compared with that of Canada, where the most prevalent practice is the institutionalization of the Rand formula, i.e. the compulsory payment of union fees, Peetz suggests that both legislative change and modifications in union practices will be necessary to right the imbalances now experienced by Australian workers.

In focusing on the impact of globalization on the Mexican economy, Arnulfo Arteaga Garcia points to the need for the emergence of new forms of citizenship. Current economic transformations have had a profound impact on both the working and living conditions of Mexican workers. Arteaga's study investigates trends in four different economic sectors. While Mexican manufacturing exhibits many of the features of modern so-called high-performance production systems, these new managerial practices do not necessarily translate into improved working conditions and greater citizenship through participation in economic decisions in the workplace. By its very nature, the growth of the informal sector, which is of huge importance in most developing economies, escapes legal regulation and is often characterized by illegal activities: it is therefore difficult to apply notions of citizenship at work to this part of the economy. This is also the case of a third economic sector - that of the fifth of the overall Mexican workforce who are migrant workers in the United States. They might be seen as a natural extension of the Mexican informal economy inasmuch as their illegal status provides an abundant supply of cheap labour for American employers. Finally, one might think that the high tech employment in the new economy corresponds to an idyll of knowledge work whereas, in fact, these workers are most often poorly paid, engaged in routine work (such as call centres) and, due to their precariousness, have little access to legal protections at work. For Arteaga, globalization therefore heralds increasing numerical and functional flexibility for Mexican workers who face both a climate of uncertainty and exclusion from basic forms of protection at work. 
Indeed, in his view, the dismantling of the social state further undermines the classic evolutionary narrative of citizenship at work, as suggested by T. H. Marshall. According to Arteaga, the solution to this state of affairs must involve a more full-blown development of global citizenship, which can only be the result of the efforts of social actors to move labour protection back to a place of privilege in the conception of the rights of citizenship and to build bridges between the demands of workers in both North and South.

Yves Hallée looks at the question of citizenship at work in terms of identity. He is interested in how dominant values shape the construction of social identities and, in particular, how they affect our recognition of women. Citizenship was, of course, constructed in reference to male roles; women were simply excluded from the equation. More current approaches offer three understandings of the role of women in society: first, a citizenship which is gender neutral, focused on the formal equality of rights and duties between men and women, and tending to ignore both a history of subordination of women and the resultant insufficiency of opportunity; second, a gendered citizenship, which is focused on values traditionally recognized as women's values, such as the ethics of solicitude and care; and, finally, a pluralist citizenship in which gender is just one avenue of analysis (as are age, social status, health, sexuality, etc.) in the construction of social identities. From this latter perspective and in relation to the question of pay equity, Hallée believes that the wage discrimination experienced by women is the result of the lesser value ascribed to caring work. He therefore makes a plea for a feminization of men's work in order that pay equity and the spread of the value of care giving and of compassion associated with women's work be given full economic and social recognition.

Georgina Murray suggests that the notion of citizenship at work can provide guiding principles to public and private policies about work. Its ability to do so, however, will depend on the legitimacy accorded to the idea of citizenship at work, notably by public decision makers. Murray's analysis leads inevitably to the conclusion that the potential for real citizenship at work is necessarily limited in a capitalist economy characterized by neo-liberalism and class politics. Her historical investigation of the different schools of thought in classical political economy suggests how such thinking places limits on the possibilities of citizenship. Whether in terms of the original theories of liberalism or their radicalization under neo-liberalism, all subscribe to the reduction of labour costs and the increase of productivity. In contrast to these liberal interpretations, John Maynard Keynes highlighted the importance of the social state, whereas Marxist critics have tended to point to the limits of social democracy in a capitalist economy. For Murray, it is clear that the underlying inequalities at the core of liberal political 
economy are in conflict with the egalitarian aspirations of citizenship at work. Her analysis points to the need to look beyond national frontiers in a global economy. Citizenship in one country is unlikely to prevail in the current context and it is therefore necessary to think in terms of a global citizenship. This should be carried forward by workers movements seeking a range of economic rights for all citizens. The notion of citizenship at work would then assume its veritable meaning.

While this brief overview of the contributions to this special issue on citizenship at work certainly underscores a diversity of approaches, we also believe that there are a number of common themes that need to be highlighted.

First, and irrespective of foolish occupational temptation on the part of people who study work and employment as a profession to want to construct coherence in the face of chaos, there is clearly in all of these contributions a search for a more comprehensive and articulated account of life at work. Citizenship at work potentially offers such coherence, though its multiple meanings and epistemological pluralism certainly give rise to caution and the increased need for rigueur in taking up this challenge.

Second, part of this search stems from the increasing disconnect between traditional models of work and their institutionalized policy frameworks on the one hand and the many changes in the world of work on the other hand. This is all the more so because so many jobs seem to be getting worse, characterized by chronic insecurity and alienation from the fruits of human agency in work (see, for example, Green, 2006), while other jobs just keep getting better, as manifested by the ability of some workplaces to attain higher degrees of organizational citizenships of all sorts. Faced with this increasing dispersion, national policy frameworks are fractured and unable to capture adequately either end of the spectrum.

Third, above and beyond the specific characteristics of these jobs, there is a need for an overarching narrative that also takes account of the characteristics of the persons likely to occupy these jobs, not least because the notion of job might just as well extend to reproductive and caring work as to firing the engines of competitive performance in key international industries. This suggests the need for new articulations between different kinds of identities: individual and collective, North and South, productive and reproductive, and men and women in different phases of their life cycles, to name just a few of these identities at play. This further raises the complex interplay between individualism and collectivism, between the individual and the group and also between status and identity; just as 
it commends us to think further about the legal and policy instruments designed to confer status and recognize identity.

Fourth, one cannot but be struck by the immense gaps between the aspirational horizons of transnational citizenship as per a transcendent narrative of decent work (World Commission, 2004) versus, as is so elegantly illustrated by the text of Arteaga in this issue, the deep paradoxes of the illegal Mexican worker in the United States working below minimum standards and unable to access minimal rights at work in the country of origin and in the country of adoption. This gap between aspiration and reality will continue to act as a motor for thinking about the perils and potentialities of citizenship at work and also the transformative potential of citizenship as a unifying project (see also Johnston, 2000).

Fifth, there is a palpable need for policy accounts that seek to build bridges between these gaps, fragmentations and tensions. All the contributors appear to agree that blind faith in the market is unlikely to lead to a fuller realization of citizenship at work. All would seem to point to the need to reconstruct some policy coherence. Yet, none really suggest that it is possible to return to points of departure as we have know them, be they in yearning for a nostalgic reconstitution of collective labour law or the reinvention of the Welfare State, not least because of significant changes in the organization of work and their relations to larger socioeconomic trends.

Sixth, one of the more telling conclusions emerging from the texts in this special issue concerns the negotiated character of citizenship at work. This is, of course, a core insight of the vision of work as a continuous process of regulation. Globalization might thus be construed as a series of opportunities for reregulating both work and its processes of regulation (Murray, Lévesque and Vallée, 2000). This highlights the need to study these ongoing processes in a variety of ways. It also points to the importance of traditional and emerging social actors. Citizenship provides one avenue of analysis for the complex re-regulation of working lives and it is also to be understood as both a work in progress and a transformative narrative for social actors seeking to bring about change at work.

Last, but not least, the veritable conceptual emporium that is this special issue of $R I / I R$ merits closer scrutiny and invites other contributors. Since this introduction barely scratches the surface, we can only commend our readers to partake of the varied and challenging contributions that follow. In so doing, we can only hope to renew at least some of the analytical challenges set out so presciently by Harry Arthurs' 1967 invitation to think about the larger dimensions of work in society and to bring the workplace and working lives into the realm of citizenship. 


\section{REFERENCES}

ARTHURS, Harry. 1967. "Developing Industrial Citizenship: A Challenge for Canada's Second Century.” Canadian Bar Review, XLV, 786-830.

ARTHURS, Harry. 1996. "Labour Law without the State." University of Toronto Law Journal, 46, 1-45.

ARTHURS, Harry. 1999. "The New Economy and the New Legality: Industrial Citizenship and the Future of Labour Arbitration." Canadian Labour and Employment Law Journal, 7 (1), 46-63.

Castells, Manuel. 1996. The Rise of the Network Society. Oxford: Blackwell Publishers.

Coutu, Michel. 2004. "Industrial Citizenship, Human Rights and the Transformation of Labour Law. A Critical Assessment of Harry Arthurs' Legalization Thesis." Revue canadienne Droit et Société/Canadian Journal of Law and Society, 19, 73-92.

FranCFORT, Isabelle, Florence Osty, Renaud SAINSAULIEU and Marc Uhalde. 1995. Les mondes sociaux de l'entreprise. Paris: Deslée de Brouwer.

Fudge, Judy and Leah Vosko. 2001. "By Whose Standards? Reregulating the Canadian Labour Market." Economic and Industrial Democracy, 22, 327-356.

Green, Francis. 2006. Demanding Work: The Paradox of Job Quality in the Affluent Economy. Princeton: Princeton University Press.

HABERMAS, Jürgen. 1996. "Citizenship and National Identity." Between Facts and Norms. Contributions to a Discourse Theory of Law and Democracy. Translation by William Regh. Cambridge: MIT Press, 491-515.

HABERMAS, Jürgen. 2000. Après l'État-nation. Une nouvelle constellation politique. Translation by Rainer Rochlitz. Paris: Fayard.

HASSENTEUFEL, Patrick. 1996. "L'État-providence ou les métamorphoses de la citoyenneté." L'Année sociologique, 46 (1), 127-149.

Held, David. 1995. Democracy and the Global Order. Cambridge: Polity Press.

Hodson, Randy. 2001. Dignity at Work. Cambridge: Cambridge University Press.

Johnston, Paul. 2000. "The Resurgence of Labor as Citizenship Movement in the New Labor Relations Environment." Critical Sociology, 26 (1-2), 139-160.

KAHN-Freund, Otto. 1954. "Legal Regulation.” The System of Labour Relations in Great Britain. A. Flanders and H. Clegg, eds. Oxford: Oxford University Press, 42-127.

Kerr, Clark, John Dunlop, Frederick Harbison and Charles Myers. 1960. Industrialism and Industrial Man. Cambridge: Harvard University Press.

Marshall, T. H. 1949. "Citizenship and Social Class." Reprint in Sociology at the Crossroads and other Essays. 1963. London: Heinemann, 67-127.

Murray, Gregor, Christian Lévesque and Guylaine Vallée. 2000. "The Re-regulation of Labour in a Global Context: Conceptual Vignettes from Canada." The Journal of Industrial Relations, 42 (2), 234-257. 
Murray, Gregor, Jacques Bélanger, Anthony GiLes and Paul-André LAPOINTE, eds. 2002. Work and Employment Relations in the High-Performance Workplace. London: Continuum.

PIORE, Michael. 1995. Beyond Individualism: How Social Demands of the New Identity Groups Challenge American and Political Life. Cambridge: Harvard University Press.

Putnam, Robert D. 2000. Bowling Alone: The Collapse and Revival of American Community. New York: Simon and Schuster.

SCHNAPPER, Dominique. 1997. Community of Citizens. Translated by Séverine Rosée. New Brunswick, N.J.: Transaction Publishers.

Schnapper, Dominique. 1997a. Contre la fin du travail. Paris: Éditions Textuel.

SCHNAPPER, Dominique. 2002. La démocratie providentielle. Essai sur l'égalité contemporaine. Paris: Gallimard.

Stasiulis, Davia K. and Abigail B. BAKAn. 2005. Negotiating Citizenship: Migrant Women in Canada and the Global System. Toronto: University of Toronto Press.

Stone, Katherine W. 2004. From Widgets to Digits: Employment Regulation for the Changing Workplace. Cambridge: Cambridge University Press.

Vallée, Guylaine, Michel Coutu and Marie-Claude Hébert. 2002. "Implementing Equality Rights in the Workplace: An Empirical Study." Canadian Labour and Employment Law Journal, 9, 71-117.

World Commission on the Social Dimension of Globalization. 2004. A Fair Globalization: Creating Opportunities for All. Geneva: International Labour Organization. 